\title{
Early practices in endonasal skull base surgery during the COVID-19 pandemic: a global survey
}

\author{
Pierre-Olivier Champagne, MD, PhD, ${ }^{1}$ Michael M. McDowell, MD, ${ }^{1}$ Eric W. Wang, MD, ${ }^{1,2}$ \\ Carl H. Snyderman, MD, ${ }^{1,2}$ Georgios A. Zenonos, MD, ${ }^{1}$ and Paul A. Gardner, MD ${ }^{1,2}$ \\ Departments of ${ }^{1}$ Neurosurgery and ${ }^{2}$ Otolaryngology, University of Pittsburgh Medical Center, Pittsburgh, Pennsylvania
}

OBJECTIVE During the severe acute respiratory syndrome coronavirus 2 (SARS-CoV-2) pandemic, endoscopic endonasal surgery (EES) is feared to be a high-risk procedure for the transmission of coronavirus disease 2019 (COVID-19). Nonetheless, data are lacking regarding the management of EES during the pandemic. The object of this study was to understand current worldwide practices pertaining to EES for skull base/pituitary tumors during the SARS-CoV-2 pandemic and provide a basis for the formulation of guidelines.

METHODS The authors conducted a web-based survey of skull base surgeons worldwide. Different practices by geographic region and COVID-19 prevalence were analyzed.

RESULTS One hundred thirty-five unique responses were collected. Regarding the use of personal protective equipment (PPE), North America reported using more powered air-purifying respirators (PAPRs), and Asia and Europe reported using more standard precautions. North America and Europe resorted more to reverse transcriptase-polymerase chain reaction (RT-PCR) for screening asymptomatic patients. High-prevalence countries showed a higher use of PAPRs. The medium-prevalence group reported lower RT-PCR testing for symptomatic cases, and the high-prevalence group used it significantly more in asymptomatic cases.

Nineteen respondents reported transmission of COVID-19 to healthcare personnel during EES, with a higher rate of transmission among countries classified as having a medium prevalence of COVID-19. These specific respondents (medium prevalence) also reported a lower use of airborne PPE. In the cases of healthcare transmission, the patient was reportedly asymptomatic $32 \%$ of the time.

CONCLUSIONS This survey gives an overview of EES practices during the SARS-CoV-2 pandemic. Intensified preoperative screening, even in asymptomatic patients, RT-PCR for all symptomatic cases, and an increased use of airborne PPE is associated with decreased reports of COVID-19 transmission during EES.

https://thejns.org/doi/abs/10.3171/2020.9.FOCUS20569

KEYWORDS COVID-19; endoscopic endonasal surgery; pituitary; skull base; survey

$\mathrm{E}$ NDOSCOPIC endonasal surgery (EES) has become widelsy accepted for many ventral skull base pathologies, ${ }^{1,2}$ providing direct access to many midline lesions. EES is an integral component of a modern skull base surgeon's armamentarium and has become the standard of care for many skull base pathologies. This standard of care is currently colliding with the worldwide spread of an upper airway virus, severe acute respiratory syndrome coronavirus 2 (SARS-CoV-2), which presents as an upper respiratory illness, is known to transmit via the upper airway, and may have a high mortality rate, especially in the elderly or those with comorbidities. ${ }^{3}$ First reported from
Wuhan, China, on December 31, 2019 (https://www.who. int/emergencies/diseases/novel-coronavirus-2019), the virus has spread rapidly and become a global pandemic. There have been anecdotal reports of the spread of coronavirus disease 2019 (COVID-19; the disease caused by the novel coronavirus) to medical professionals during endonasal surgery, and surgical societies have developed guidelines for surgery during the pandemic. ${ }^{4}$ However, there remains a dearth of data to understand the danger of such surgery in a wide variety of patient populations.

A high risk for disease transmission is likely with symptomatic patients, especially when the sinuses or na-

ABBREVIATIONS COVID-19 = coronavirus disease 2019; EES = endoscopic endonasal surgery; PAPR = powered air-purifying respirator; PPE = personal protective equipment; RT-PCR = reverse transcriptase-polymerase chain reaction; SARS-CoV-2 = severe acute respiratory syndrome coronavirus 2.

SUBMITTED June 22, 2020. ACCEPTED September 18, 2020.

INCLUDE WHEN CITING DOI: 10.3171/2020.9.FOCUS20569. 
sopharyngeal tissues are transgressed. Workman et al. found that the high-speed drill creates the most spray outside of the nasal cavity. ${ }^{4}$ Protecting surgeons and associated professionals without compromising the quality of care is a difficult balance during this pandemic. The risk of transmission in asymptomatic patients is likely much lower, though there is even less information regarding the number of asymptomatic carriers, their risk of transmission, and any necessary precautions. The risk associated with asymptomatic patients is likely affected by many factors, including symptom screening and regional prevalence.

Safe endonasal surgery likely requires a balance among preoperative screening, preoperative testing, and intraoperative personal protective equipment (PPE). Understanding current practices across the globe and attempting to gauge their efficacy in regions of varying prevalence is one starting point in creating guidelines for endonasal surgery in such a unique and difficult time. The survey presented in this paper is intended to provide such a baseline from which to begin the discussion and development of evidence-based guidelines.

\section{Methods}

A web-based survey of skull base surgeons across the globe was sent via SkullBaseCongress.com, a free educational website and online community for skull base surgeons of all specialties (Supplemental Material). Respondents were asked to identify their region of practice and were then questioned about their current practice policies surrounding EES. The survey focused on surgical indication, preoperative screening, use of PPE, and COVID-19 infection of healthcare professionals from exposure during EES.

\section{Analysis by COVID-19 Prevalence in Each Country}

An analysis was performed by dividing the survey sample into 3 groups based on the COVID-19 prevalence in each respondent's country. To create these groups, the total number of confirmed COVID-19 cases in each represented country was retrieved from the World Health Organization Situation Report on April 18, 2020..$^{5}$ This case count was divided by the country population to obtain the prevalence per million inhabitants. The survey sample was then divided into 3 groups according to prevalence/ million inhabitants. The low-prevalence group included countries from the 1 st to the $33 \mathrm{rd}$ percentiles inclusive, the medium-prevalence group included countries from the 34th to the 65th percentiles inclusive, and the high-prevalence group included countries in the 66th to the 100th percentiles inclusive.

\section{Statistical Analysis}

Statistical analysis was conducted using SPSS software version 24.0 (IBM Corp.). A univariate 2-tailed chi-square test was used for nominal and ordinal variables, and Fisher's exact test was used when the sample was inadequate for chi-square analysis. When comparing more than two variables, the Freeman-Halton extension of Fisher's exact test for multiple comparisons was used. Post hoc analysis
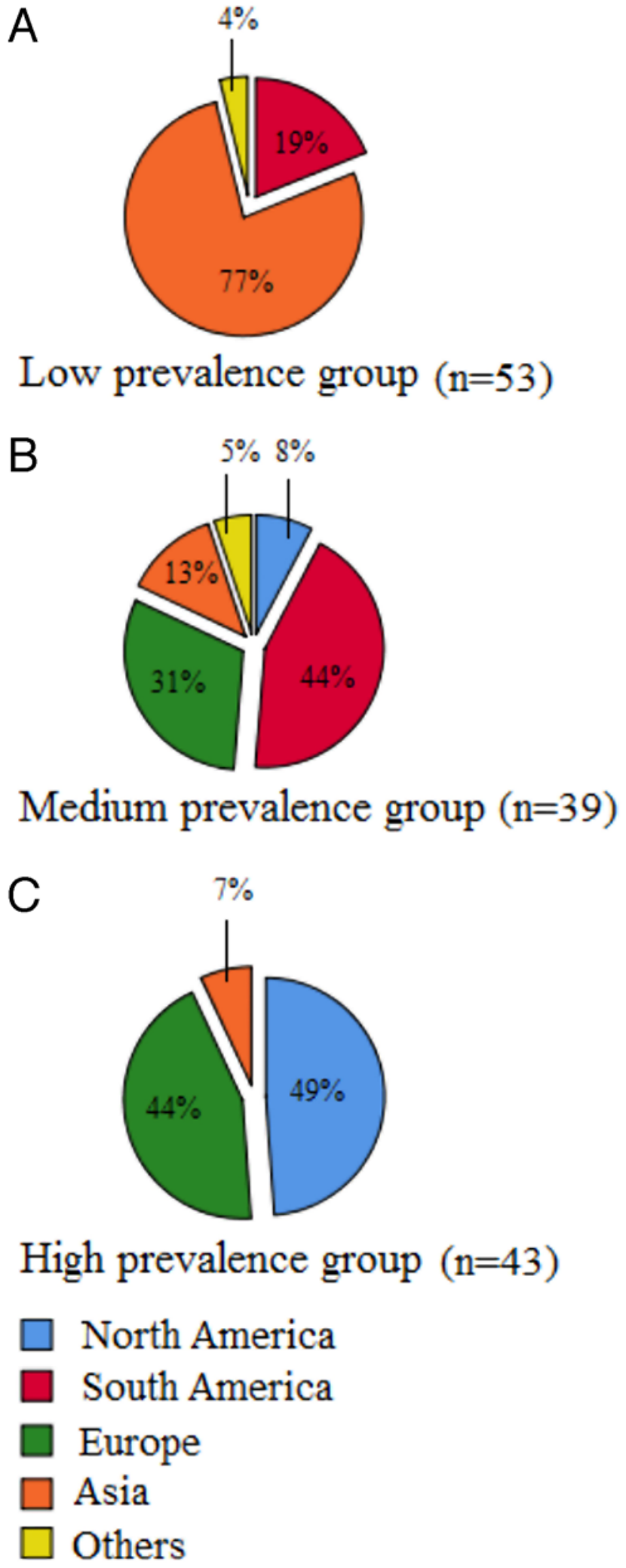

FIG. 1. Distribution of geographic regions among prevalence groups. 


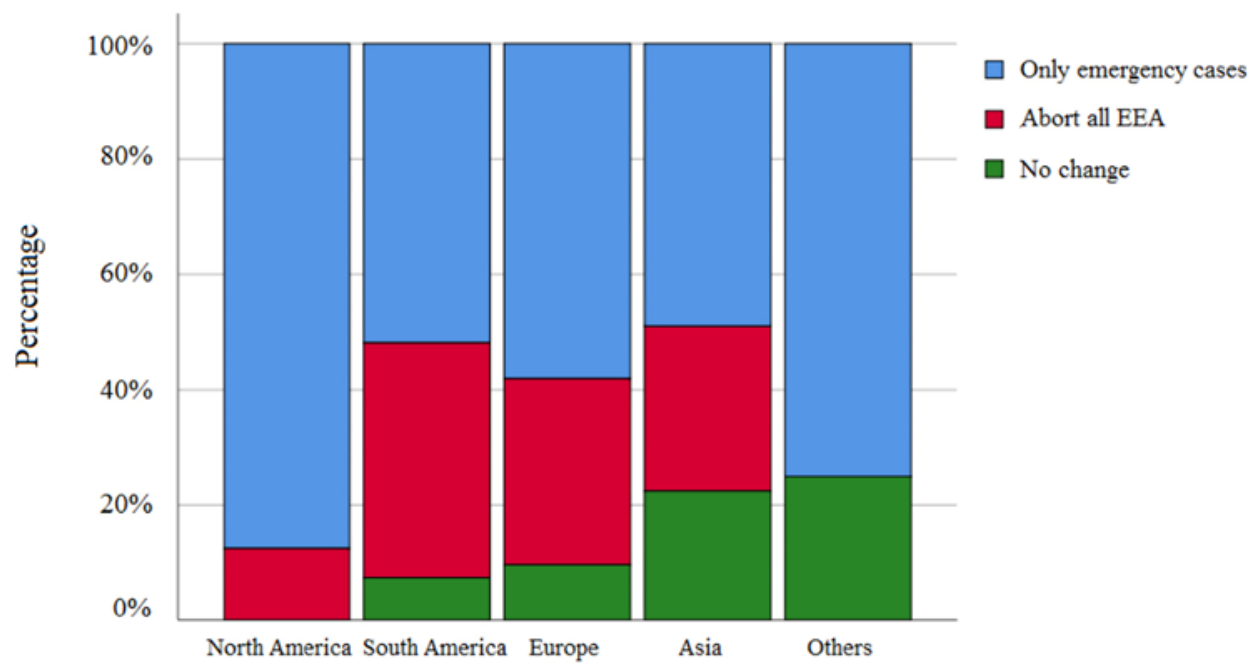

FIG. 2. Changes in EES policy. EEA = endoscopic endonasal approach.

using the residual comparison method ${ }^{6}$ was performed as needed to help determine between which variables of the chi-square test there was a statistically significant difference. For all tests, a p value $<0.05$ was considered significant.

\section{Results}

\section{Surveyed Population}

A total of 135 unique responses were collected from six continents. Forty-nine (36.3\%) responses were received from Asia; 31 (23.0\%) from Europe; 27 (20.0\%) from Central America, South America, and the Caribbean (grouped together as South America); 24 (17.8\%) from North America; 2 (1.5\%) from Africa; and 2 (1.5\%) from Australia. Sixty-eight (50.4\%) respondents were neurosurgeons, $65(48.1 \%)$ were otolaryngologists, $1(0.7 \%)$ was an oral maxillofacial surgeon, and $1(0.7 \%)$ was a nonphysician responding on behalf of both neurosurgery and otolaryngology.

\section{COVID-19 Prevalence}

The low-prevalence group $(\mathrm{n}=53)$ consisted of countries having a prevalence $\leq 137.4 /$ million inhabitants, the medium-prevalence group $(\mathrm{n}=39)$ comprised countries with a prevalence between 137.4 and 1485/million inhabitants, and the high-prevalence group $(n=43)$ comprised countries having a prevalence $\geq 1485 /$ million inhabitants. Asia made up $77.4 \%$ of the low-prevalence group; South America and Europe, $44 \%$ and $31 \%$ of the medium-prevalence group, respectively; and North America and Europe, the majority of the high-prevalence group (49\% and $44 \%$, respectively; Fig. 1).

\section{Change in EES Policy}

Eighty $(59.3 \%)$ respondents reported proceeding with EES only in settings of impending neurological or visual compromise. Seventeen (12.6\%) reported no change in EES policies, and 38 (28.1\%) reported a complete cessa- tion of EES (Fig. 2). North American respondents reported the highest rate of policies for continuing only emergency cases compared to other regions ( $p=0.002$, Fisher's exact test). Asia had a significantly higher rate of not changing their EES policy than the other countries $(22.4 \%$ vs $0 \%$, 7.4\%, and $9.7 \%$ for North America, South America, and Europe, respectively, $\mathrm{p}=0.009$, Fisher's exact test).

\section{Use of Intraoperative PPE}

In terms of additional PPE during EES (regardless of COVID status), 30 (22.2\%) respondents reported using standard precautions (nonairborne masks), 86 (63.7\%) reported using facemasks and N95 masks, 8 (5.9\%) reported using N95 masks alone, and 11 (8.1\%) reported the use of powered air-purifying respirators (PAPRs; Table 1). The use of PAPRs was significantly more prevalent in North America than in other regions ( $p=0.012$, Fisher's exact test). Standard precautions were more frequently used in Asia and Europe than in other regions $(32.7 \%$ and $38.7 \%$, respectively; $\mathrm{p}=0.027$ and 0.012 , respectively, Fisher's exact test). In terms of anesthesia and ancillary staff use of PPE, 76 (56.3\%) respondents reported the use of N95 masks and face shields, 41 (30.4\%) reported the use of standard equipment, 10 (7.4\%) used N95 masks alone, and 8 (5.9\%) used PAPR. Standard equipment was used more frequently in Europe and Asia than in other regions (45\% and $43 \%$, respectively; $\mathrm{p}=0.045$ and 0.016 , respectively, Fisher's exact test).

Although the relationship was not statistically significant, we noted a trend toward the increased use of regular protection by ancillary personnel compared to that by surgeons in Asia (41.7\% vs $32.7 \%$, respectively, $p=0.361$ ) and in Europe (45.2\% vs 38.7\%, respectively, $\mathrm{p}=0.315$ ).

Regarding an analysis by COVID-19 prevalence, the high-prevalence group showed a higher use of PAPR $(16.3 \%$ vs $2.6 \%$ and $5.7 \%$ in the medium- and low-prevalence groups, respectively, $\mathrm{p}=0.016$ ) and a lower use of regular protection equipment $(9.3 \%$ vs $28.2 \%$ and $28.3 \%$, respectively, $\mathrm{p}=0.012$ ). 
TABLE 1. Use of intraoperative PPE among geographic regions and prevalence groups

\begin{tabular}{|c|c|c|}
\hline Variable & No. (\%) & $\mathrm{p}$ Value \\
\hline \multicolumn{3}{|l|}{ Use of PPE during EES } \\
\hline N95 \& facemask & $86(64)$ & \multirow{4}{*}{ - } \\
\hline N95 alone & $8(6)$ & \\
\hline PAPR & $11(8)$ & \\
\hline Standard precautions & $30(22)$ & \\
\hline \multicolumn{3}{|l|}{ Use of N95 \& facemask } \\
\hline North America & $18(75)$ & \multirow{5}{*}{0.046} \\
\hline South America & $22(81)$ & \\
\hline Europe & $15(48)$ & \\
\hline Asia & $28(57)$ & \\
\hline Others & $3(75)$ & \\
\hline \multicolumn{3}{|l|}{ Use of N95 alone } \\
\hline North America & $0(0)$ & \multirow{5}{*}{0.162} \\
\hline South America & $4(15)$ & \\
\hline Europe & $2(6)$ & \\
\hline Asia & $2(4)$ & \\
\hline Others & $0(0)$ & \\
\hline \multicolumn{3}{|l|}{ Use of PAPR } \\
\hline North America & $5(21)$ & \multirow{5}{*}{0.012} \\
\hline South America & $1(4)$ & \\
\hline Europe & $2(6)$ & \\
\hline Asia & $3(6)$ & \\
\hline Others & $0(0)$ & \\
\hline \multicolumn{3}{|l|}{ Use of standard precautions } \\
\hline North America & $1(4)$ & \multirow{5}{*}{0.027} \\
\hline South America & $0(0)$ & \\
\hline Europe & $12(39)$ & \\
\hline Asia & $16(33)$ & \\
\hline Others & $1(25)$ & \\
\hline \multicolumn{3}{|l|}{ Use of N95 \& facemask } \\
\hline Low-prevalence group & $33(62)$ & \multirow{3}{*}{0.317} \\
\hline Medium-prevalence group & $23(59)$ & \\
\hline High-prevalence group & $30(70)$ & \\
\hline \multicolumn{3}{|l|}{ Use of N95 alone } \\
\hline Low-prevalence group & $2(4)$ & \multirow{3}{*}{0.162} \\
\hline Medium-prevalence group & $4(10)$ & \\
\hline High-prevalence group & $2(5)$ & \\
\hline \multicolumn{3}{|l|}{ Use of PAPR } \\
\hline Low-prevalence group & $3(6)$ & \multirow{3}{*}{0.016} \\
\hline Medium-prevalence group & $1(3)$ & \\
\hline High-prevalence group & $7(16)$ & \\
\hline \multicolumn{3}{|l|}{ Use of standard precautions } \\
\hline Low-prevalence group & $15(28)$ & \multirow{3}{*}{0.012} \\
\hline Medium-prevalence group & $11(28)$ & \\
\hline High-prevalence group & $4(9)$ & \\
\hline
\end{tabular}

Boldface type indicates statistical significance.

\section{Screening in Symptomatic and Asymptomatic Patients}

For patients presenting with fever, cough, and other findings concerning for symptomatic COVID-19 infection, responders reported using the following measures: RT-PCR testing, 103 (76.3\%); preoperative quarantine, 48 (35.6\%); lung CT, 50 (37.0\%); screening questionnaires, 76 (56.3\%); temperature measurements, 82 (60.7\%); viral cultures, 18 (13.3\%); cancellation of all interventions for the patient, 15 (11.1\%); and no screening before surgery, 2 (1.5\%; 1 respondent in South America and 1 in Asia). North and South American respondents were significantly less likely to report the use of lung CT ( $p=0.049$, Fisher's exact test) or temperature screening ( $p=0.013$, Fisher's exact test) for symptomatic patients (Fig. 3). North American respondents were also significantly less likely to report the use of questionnaires to screen symptomatic patients ( $p=0.001$, Fisher's exact test). There was a statistically significant difference between prevalence groups with respect to RT-PCR, lung CT use, and surgery avoidance. RT-PCR use was significantly lower in the medium-prevalence group than in the other two groups $(61.5 \%$ vs $86.8 \%$ and $76.7 \%$ for the low- and high-prevalence groups, respectively, $p=0.023$; Fig. 4). The lowprevalence group had a higher use of preoperative lung CT than the other two groups $(49.1 \%$ vs $23.1 \%$ and $34.9 \%$ for the medium- and high-prevalence groups, respectively, $p=0.040$ ) and also had a higher rate of avoiding surgery in symptomatic patients $(18.9 \%$ vs $10.3 \%$ and $2.3 \%$, respectively, $\mathrm{p}=0.033$ ).

For patients without symptoms of or known exposure to COVID-19, 61 (45.2\%) respondents reported the use of RT-PCR testing prior to surgery, $30(22.2 \%)$ reported a preoperative quarantine policy, $26(19.3 \%)$ reported performing lung CT, 99 (73.3\%) reported questionnaire use, $89(65.9 \%)$ reported temperature screening, $11(8.1 \%)$ reported viral culture screening, $3(2.2 \%)$ reported not operating, and $6(4.4 \%)$ reported not screening in asymptomatic patients (Fig. 3). North American respondents were less likely to use lung CT for screening ( $p=0.004$, Fisher's exact test). In the prevalence analysis, there was a statistically significant difference in the use of RT-PCR, lung CT, screening questionnaire, and temperature check among groups. With regard to RT-PCR, the high-prevalence group used it significantly more often $(60.5 \%$ vs $43.6 \%$ and $34.0 \%$ in the medium- and low-prevalence groups, respectively, $\mathrm{p}=0.034$ ), the opposite of what was observed in symptomatic patients. Lung CT was used more often in the low-prevalence group (37.7\% vs $5.1 \%$ and $9.3 \%$ in the medium- and high-prevalence groups, respectively, $\mathrm{p}<$ $0.001)$. Screening questionnaires and temperature checks for asymptomatic patients were used more in the lowprevalence group $(\mathrm{p}=0.041$ and 0.031 , respectively).

\section{Transmission of COVID-19 From EES}

COVID-19 transmission to a healthcare worker from exposure during EES was reported by 19 respondents (14.1\%). The rate of transmission reported across geographic regions showed a trend, with South America and Europe having more cases, although the difference from other countries was not statistically significant (Table 2). Differences in the rate of nosocomial transmission of 

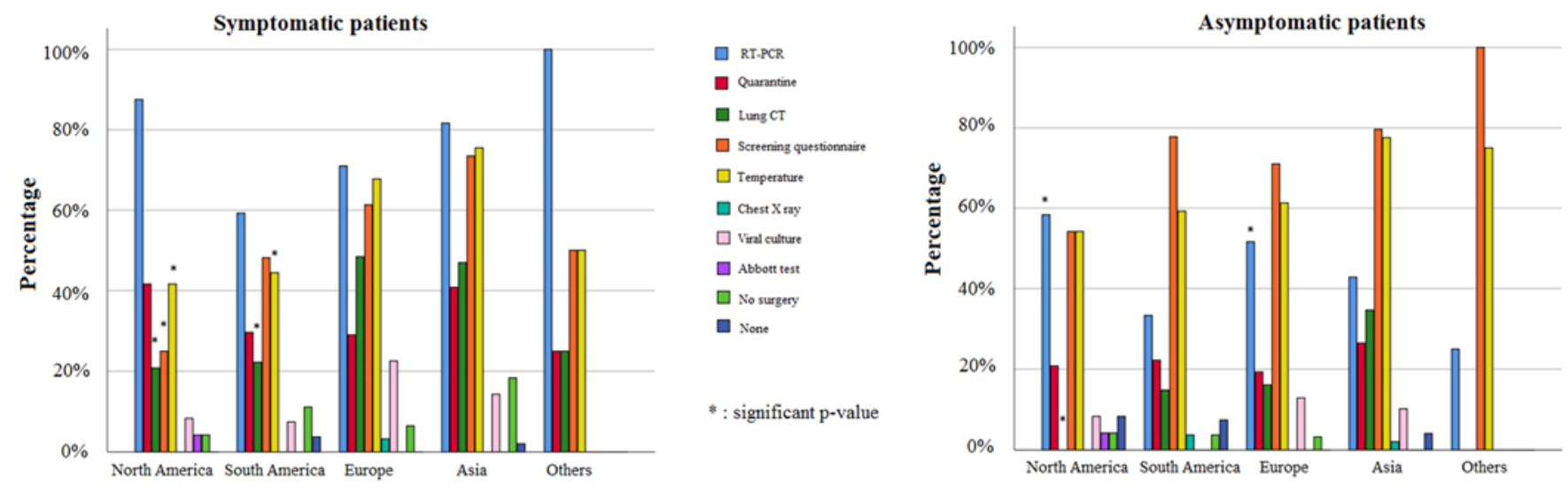

FIG. 3. Screening frequency in symptomatic and asymptomatic patients by region. Significant $p$ values represent differences in the same screening parameter compared to other regions.

COVID-19 to personnel during EES was statistically significant among the three prevalence groups, with the medium-prevalence group having a higher rate of transmission than the other two groups $(25.6 \%$ vs $7.5 \%$ and $11.6 \%$ for the low- and high-prevalence groups, respectively, $\mathrm{p}=$ 0.049 ).

Of these 19 reports, the patients were symptomatic in $13(68.4 \%)$ cases. The rate of symptomatic patients associated with these cases of transmission was not different among regions or prevalence groups. Regarding precautions taken in these 19 cases, 11 (57.9\%) reported the use of N95 masks, $4(21.1 \%)$ reported the use of PAPR, $2(10.5 \%)$ performed preoperative testing, and $2(10.5 \%)$ used no specific precautions. All the patients with whom PAPR was used were symptomatic (4), and all patients in whom preoperative testing was used (2) were asymptomatic. When these 19 patients were analyzed by COVID-19 prevalence, the medium-prevalence group did not use any PAPR ( $0 \%$ vs $50 \%$ and $40 \%$ for the low- and high-prevalence groups, respectively, $\mathrm{p}=0.034$ ) and took no specific precautions in $20 \%$ of cases (i.e., preoperative testing or airborne PPE; $20 \%$ vs $0 \%$ and $0 \%$ in the low- and highprevalence groups, respectively, $\mathrm{p}=0.034 ;$ Fig. 5).

\section{Discussion}

The SARS-CoV-2 viral load is highest in the oral and nasal cavities, ${ }^{7}$ and the common use of powered instruments that can aerosolize secretions ${ }^{8,9}$ during endoscopic endonasal skull base and pituitary procedures may make these procedures higher risk for intraoperative virus transmission to medical personnel. This has created significant anxiety among surgeons, nurses, and anesthesiologists, but there is a paucity of data on the actual risk and appropriate management of EES in that context. In cases for which the endonasal route represents the most favored corridor, prioritizing those cases that would benefit from that approach in the context of the pandemic remains challenging. ${ }^{10}$ The place of elective and urgent cases remains one of the main controversies in this context, ${ }^{11}$ and recent guidelines from the pituitary society highlighted the risk of transmission to healthcare workers during EES as one of the main challenges of pituitary surgery during the pandemic and also stressed the current paucity of data regarding EES.12 Our study aims to describe common practices and provide a basis on which to construct recommendations for the management of EES in the setting of the COVID-19 pandemic.
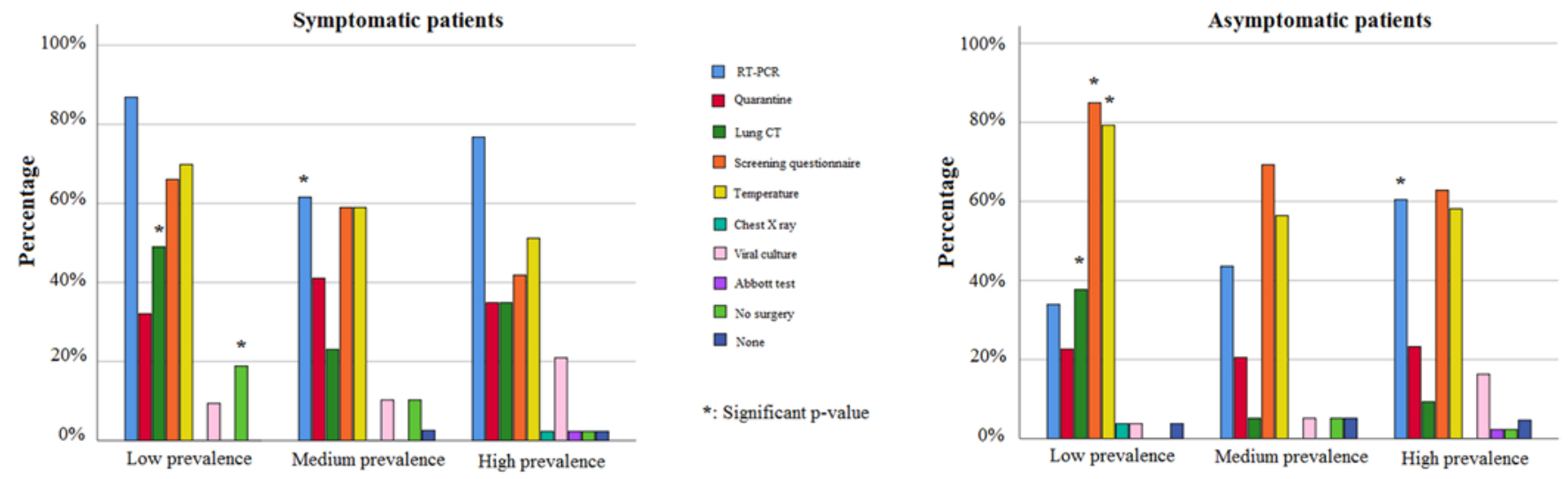

FIG. 4. Screening frequency in symptomatic and asymptomatic patients by prevalence group. Significant $p$ values represent differences in the same screening parameter compared to other prevalence groups. 
TABLE 2.Transmission of COVID-19 from EES

\begin{tabular}{|c|c|c|}
\hline Variable & No. $(\%)$ & p Value \\
\hline Incidence in surveyed population & $19(14)$ & - \\
\hline \multicolumn{3}{|l|}{ Incidence by geographic region } \\
\hline North America & $1(4)$ & \multirow{4}{*}{0.071} \\
\hline South America & $7(26)$ & \\
\hline Europe & $7(23)$ & \\
\hline Asia & $4(8)$ & \\
\hline \multicolumn{3}{|l|}{ Incidence by prevalence group } \\
\hline Low prevalence & $4(8)$ & \multirow{3}{*}{0.049} \\
\hline Medium prevalence & $10(26)$ & \\
\hline High prevalence & $5(12)$ & \\
\hline \multicolumn{3}{|l|}{ Respondent's speciality } \\
\hline Neurosurgery & $10(15)$ & \multirow{3}{*}{0.672} \\
\hline ENT & $8(13)$ & \\
\hline Other & $1(20)$ & \\
\hline Rate of symptomatic patients & $13(68)$ & - \\
\hline \multicolumn{3}{|c|}{ Symptomatic patients by geographic region } \\
\hline North America & $1(100)$ & \multirow{4}{*}{0.467} \\
\hline South America & $6(86)$ & \\
\hline Europe & $3(43)$ & \\
\hline Asia & $3(75)$ & \\
\hline \multicolumn{3}{|c|}{ Symptomatic patients by prevalence group } \\
\hline Low prevalence & $3(75)$ & \multirow{3}{*}{0.99} \\
\hline Medium prevalence & $7(70)$ & \\
\hline High prevalence & $3(60)$ & \\
\hline \multicolumn{3}{|c|}{ Precautions used in cases of transmission } \\
\hline N95 mask & $11(58)$ & \\
\hline PAPR & $4(21)$ & \\
\hline Preop testing & $2(11)$ & \\
\hline No precautions & $2(11)$ & \\
\hline
\end{tabular}

ENT $=$ ear, nose, and throat.

Our survey revealed heterogenous practices among geographic regions in terms of management of EES during the COVID-19 pandemic. Commonalities that represent some degree of standard practice include preoperative RT-PCR testing for symptomatic patients, increased used of airborne PPE, and consistent use of airborne PPE for all personnel involved with the patient intraoperatively. Among the most important discrepancies are the type of intraoperative PPE available, with PAPR being used rarely and mainly in North America, and standard (nonairborne) protection with no specific measures being reported by $38 \%$ of the respondents from Europe. Although not statistically significant, there was a trend toward a greater use of standard protection among anesthesia and ancillary staff (compared to surgeons) in Europe and Asia. In the absence of evidence of a "risk-free zone" farther away from the surgical field, all operating room personnel should have the same PPE. This is especially true for anesthesia staff, considering that endotracheal intubation/extubation is considered a high-risk aerosolproducing procedure..$^{13}$
Another variable showing significant geographic heterogeneity was the preoperative screening and testing of symptomatic and asymptomatic patients. There is a tremendous amount of anxiety among surgeons and other healthcare workers surrounding the possibility of transmission of COVID-19 from asymptomatic carriers of the virus. Rates of asymptomatic disease range from $<1 \%$ in the general population of Iceland ${ }^{14}$ to an estimated rate of $17.9 \%$ in a high-prevalence group of the Princess cruise ship cohort. ${ }^{15}$ In this survey, $14 \%$ of respondents reported seeing EES transmission, and a third of these cases involved asymptomatic patients. This reinforces the need for more intense screening of asymptomatic patients undergoing EES to ensure that they are indeed asymptomatic. It also supports the use of preoperative testing in asymptomatic patients.

\section{Subgroup Analysis Related to COVID-19 Prevalence}

The results of the analysis based on COVID-19 prevalence revealed discrepancies among the groups regarding the screening of symptomatic and asymptomatic patients. For symptomatic patients, we could explain the decreased use of RT-PCR in the medium-prevalence group and the increased surgical avoidance in the low-prevalence group as reflections of local resources. Among asymptomatic patients, the greater use of screening questionnaires and temperature measurement in the low-prevalence group in contrast to the higher use of RT-PCR in the high-prevalence group follows the local prevalence of the disease, with higher-prevalence regions screening more aggressively.

Interestingly, there was a higher rate of reported transmission of COVID-19 to healthcare personnel from EES in the medium-prevalence group. One would assume that the highest rate of transmission would have occurred in the high-prevalence group, but other data from this survey could help to explain this finding. The medium-prevalence group had a lower rate of RT-PCR testing for screening symptomatic patients, which could have led to an underdiagnosis at the time of surgery. The high-prevalence group had an appropriately increased rate of RT-PCR in asymptomatic patients, which could also play a role in these transmission rates. In addition, the use of intraoperative PAPR was higher in the high-prevalence group, hinting that the use of PAPR could help to better protect against COVID-19 transmission. This is supported by the fact that in the specific cases in which transmission was documented, the medium-prevalence group was the only one in which the respondents reported transmission but also reported no use of PAPR and in some instances no use of any specific measures (Fig. 5).

These data could support the use of PAPR during surgery as well as the notion that N95 masks may be less protective than PAPR, with some evidence suggesting that N95 masks do not offer protection superior to that of a regular surgical mask..$^{16}$

There are a few recommendations to be considered based on this survey. Chiefly, they include increased testing and consistent PPE use when possible; strengthening of screening, including temperature measurement and questionnaires; and a consideration of preoperative quarantining of patients undergoing EES. The overall low risk 
A
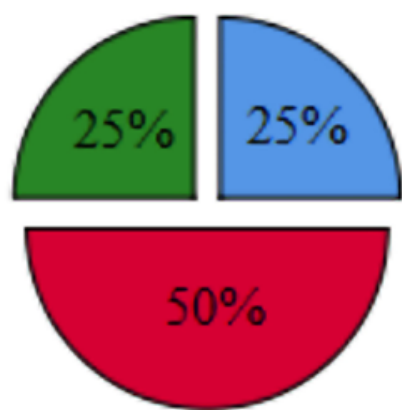

\section{Low prevalence $(n=4)$}
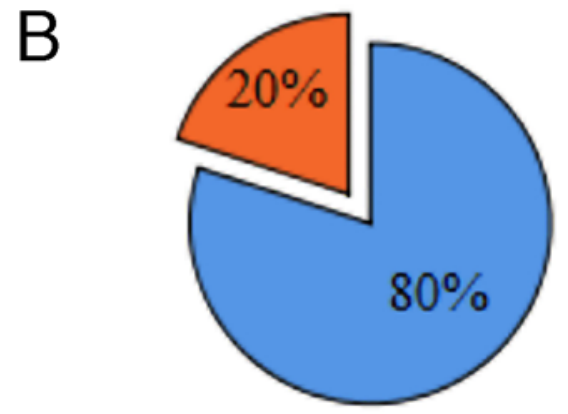

\section{Medium prevalence $(n=10)$}

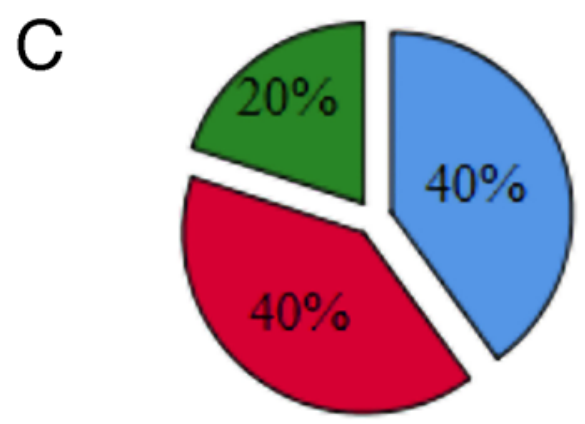

\section{High prevalence $(n=5)$}

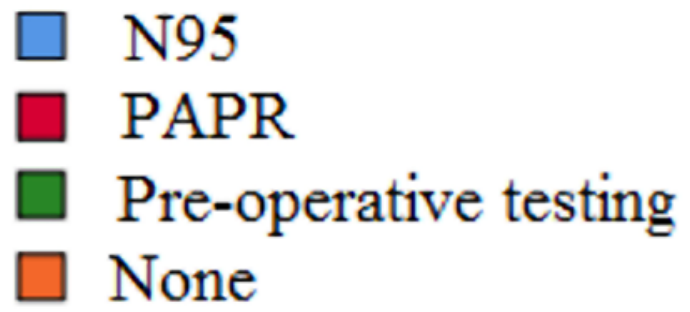

FIG. 5. Use of PPE and screening among 19 cases of transmission of COVID-19 to healthcare personnel during EES. None $=$ no precautions (i.e., preoperative testing or airborne PPE). of transmission to healthcare personnel captured by this survey and the practices associated with the survey suggest that consistent use of these practices described herein may help limit this risk and the associated fear even more. This topic clearly needs much more rigorous study, but it is hoped that this paper among others will help provide the impetus for stronger data and a better understanding of the risks associated with operating on many patients who would benefit from endonasal surgery and have pathologies whose treatment cannot be reasonably delayed despite the risk to those caring for them.

\section{Study Limitations}

The current study is subject to selection bias from its design. Recall bias could also have affected the respondents. The size of the survey sample is relatively small, but the survey was addressed to a very specific population; thus, the sample is likely representative. This study also did not account for the stage of disease (relative to peak infection rates) that specific countries or regions were experiencing. This could impact the results for groups with different prevalences. For example, the low-prevalence group was largely composed of Asian countries, which were among the first to be impacted and therefore potentially more established in their response. In addition, any inferences about the efficacy of policies or practices should be taken lightly given the innumerable complicating factors that could affect their impact.

\section{Conclusions}

The current survey gives an overview of the different practices applied worldwide for EES during the COVID-19 pandemic. Intensified preoperative screening, even in asymptomatic patients, coupled with an increased use of airborne PPE, is associated with decreased reports of COVID-19 transmission during EES. This suggests that more intense screening of asymptomatic patients and the use of PPE such as PAPR during surgery could help to diminish the risk of transmission, but this requires significant further study.

\section{References}

1. Hardesty DA, Prevedello DM. What are the limits of endoscopic endonasal approaches to the skull base? J Neurosurg Sci. 2018;62(3):285-286.

2. Kasemsiri P, Carrau RL, Ditzel Filho LF, et al. Advantages and limitations of endoscopic endonasal approaches to the skull base. World Neurosurg. 2014;82(6)(suppl):S12-S21.

3. Liu K, Chen Y, Lin R, Han K. Clinical features of COVID-19 in elderly patients: a comparison with young and middle-aged patients. J Infect. 2020;80(6):e14-e18.

4. Workman AD, Welling DB, Carter BS, et al. Endonasal instrumentation and aerosolization risk in the era of COVID-19: simulation, literature review, and proposed mitigation strategies. Int Forum Allergy Rhinol. 2020;10(7):798-805.

5. World Health Organization. Coronavirus disease 2019 (COVID-19): Situation report-89. Published April 18, 2020. Accessed October 15, 2020. https://www.who.int/docs/ default-source/coronaviruse/situation-reports/20200418sitrep-89-covid-19.pdf?sfvrsn=3643dd38_2

6. Sharpe D. Chi-square test is statistically significant: now what? Pract Assess Res Eval. 2015;20(8):1-10. 
7. Wang W, Xu Y, Gao R, et al. Detection of SARS-CoV-2 in different types of clinical specimens. JAMA. 2020;323(18) $1843-1844$.

8. Meng L, Qiu H, Wan L, et al. Intubation and ventilation amid the COVID-19 outbreak: Wuhan's experience. Anesthesiology. 2020;132(6):1317-1332.

9. Polednik B. Aerosol and bioaerosol particles in a dental office. Environ Res. 2014;134:405-409.

10. Kolias A, Tysome J, Donnelly N, et al. A safe approach to surgery for pituitary and skull base lesions during the COVID-19 pandemic. Acta Neurochir (Wien). 2020;162(7):1509-1511.

11. Hanrahan JG, Burford C, Adegboyega G, et al. Early responses of neurosurgical practice to the coronavirus disease 2019 (COVID-19) pandemic: a rapid review. World Neurosurg. 2020;141:e1017-e1026.

12. Fleseriu M, Buchfelder M, Cetas JS, et al. Pituitary society guidance: pituitary disease management and patient care recommendations during the COVID-19 pandemic - an international perspective. Pituitary. 2020;23(4):327-337.

13. Zuo M-Z, Huang Y-G, Ma W-H, et al. Expert recommendations for tracheal intubation in critically ill patients with noval coronavirus disease 2019. Chin Med Sci J. 2020;35(2):105-109.

14. Gudbjartsson DF, Helgason A, Jonsson H, et al. Spread of SARS-CoV-2 in the Icelandic population. $N$ Eng. J Med. 2020;382:2302-2315.

15. Mizumoto K, Kagaya K, Zarebski A, Chowell G. Estimating the asymptomatic proportion of coronavirus disease 2019 (COVID-19) cases on board the Diamond Princess cruise ship, Yokohama, Japan, 2020. Euro Surveill. 2020;25(10):2000180.

16. Bartoszko JJ, Farooqi MAM, Alhazzani W, Loeb M. Medical masks vs N95 respirators for preventing COVID-19 in healthcare workers: a systematic review and meta-analysis of randomized trials. Influenza Other Respir Viruses. 2020; 14(4):365-373.

\section{Disclosures}

Dr. Snyderman is a consultant for SPIWay LLC and receives royalties from Peter Lazic US Inc. Dr. Gardner is a consultant for SPIWay LLC, Peter Lazic US Inc., and Stryker, receives royalties from Peter Lazic US Inc., and has direct stock ownership in Renerva.

\section{Author Contributions}

Conception and design: Gardner, Wang, Snyderman, Zenonos. Acquisition of data: Champagne. Analysis and interpretation of data: Gardner, Champagne, McDowell. Drafting the article: Gardner, Champagne, McDowell, Wang, Snyderman. Critically revising the article: all authors. Reviewed submitted version of manuscript: all authors. Approved the final version of the manuscript on behalf of all authors: Gardner. Statistical analysis: Champagne, McDowell, Zenonos. Administrative/technical/material support: Gardner. Study supervision: Gardner.

\section{Supplemental Information}

Online-Only Content

Supplemental material is available online.

Supplemental Material. https://thejns.org/doi/suppl/10.3171/ 2020.9.FOCUS20569.

\section{Correspondence}

Paul A. Gardner: University of Pittsburgh Medical Center, Pittsburgh,PA.gardpa@upmc.edu. 